${ }^{1}$ Centro de Excelencia en

Medicina Traslacional, CEMTBIOREN, Universidad de La

Frontera. Temuco, Chile.

${ }^{2}$ Centro de Investigación en

Epidemiología Cardiovascular y Nutricional, EPICYN, Universidad de La Frontera. Temuco, Chile.

${ }^{3}$ Departamento de Medicina Interna, Universidad de La

Frontera. Temuco, Chile.

${ }^{4}$ Departamento de Educación Física, Deportes y Recreación, Universidad de La Frontera. Temuco, Chile.

${ }^{5}$ Becado de Pediatría, Facultad de Medicina, Universidad de La Frontera. Temuco, Chile.

${ }^{6}$ Departamento de Enfermería, Universidad de La Frontera. Temuco, Chile. 'Departamento de Ciencias Básicas, Universidad de La Frontera. Temuco, Chile. ${ }^{a}$ Kinesiólogo.

bEnfermero, Mg® en Ciencias Médicas.

‘Kinesiólogo, Mg en Educación Física.

dBioquímico, PhD@ en Biología Celular y Molecular Aplicada.

EEstudiante de Tecnología Médica. †Tecnólogo Médico. gPhD.

Trabajo finaciado por el Fondo de Investigación UNETE (Proyecto \# UNT15-004) del Convenio de Desempeño Regional, FRO 1301,

Universidad de La Frontera y Proyecto FONDECyT 11150445

Recibido el 31 de marzo de 2018, aceptado el 14 de agosto de 2018.

Correspondencia a: Alvaro Cerda, PhD. Departamento de Ciencias Básicas Universidad de La Frontera Centro de Excelencia en Medicina Traslacional.

Av. Alemania 0458. Temuco, Chile. alvaro.cerda@ufrontera.cl

\section{Alta prevalencia de trastornos nutricionales por exceso, resistencia insulínica y síndrome metabólico en escolares de la comuna de Carahue, Región de la Araucanía}

\author{
JORGE SAPUNAR ${ }^{1,2,3, \mathrm{~g}, \text { NICOLÁS AGUILAR-FARÍAS }}{ }^{2,4, a}$, \\ JUAN NAVARRO ${ }^{5}$, GUSTAVO ARANEDA ${ }^{1,6, b}$, \\ DAMIAN CHANDIA-POBLETE ${ }^{4, c}$, VÍCTOR MANRÍQUEZ ${ }^{1, \mathrm{~d}}$, \\ ROBERTO BRITO $^{1, \mathrm{e}}$, ALVARO CERDA $^{1,2,7, \mathrm{f,g}}$
}

\section{High prevalence of overweight, obesity, insulin resistance and metabolic syndrome in rural children and adolescents}

Background: Childhood and adolescent obesity is a major public health problem in Chile. Aim: To characterize cardiometabolic risk factors in a population of schoolchildren from Carahue, Chile. Material and Methods: Cross-sectional assessment of 208 children aged $10.4 \pm 1.0$ years (106 women). A clinical evaluation was carried out including pubertal development according to Tanner and anthropometric parameters. A fasting blood sample was obtained to measure glucose, insulin and lipid profile. HOMA-IR and Quicki indices were calculated. Insulin resistance (IR) was established according to Burrows criteria and Barja criteria, previously proposed for the Chilean pediatric population. The metabolic syndrome (MetS) was established using the modified Cook criteria. Results: Thirty eight percent of children had overweight and $33.1 \%$ obesity. MetS was only observed in obese subjects and the frequency in this subgroup was 38\%. The prevalence of IR was $51 \%$ according to the Burrows criteria and $19 \%$ according to Barja criteria. It was more common in participants who were overweight, obese or had abdominal obesity. Children with insulin resistance according to Barja criteria, had worse anthropometric measures than their counterparts without resistance. When Burrows criteria was used, no differences in anthropometric measures were observed between participants with or without resistance. The frequency of MetS was 26 and 18\% in children with insulin resistance according to Barja and Burrows criteria, respectively. Insulin levels and insulin sensitivity indexes were positively correlated with anthropometric parameters. Conclusions: There was a high prevalence of overweight, obesity and MetS in these participants. Our results suggest that the IR criteria according to Barja allows to identify cases with higher metabolic risk.

(Rev Med Chile 2018; 146: 978-986)

Key words: Insulin Resistance; Metabolic Syndrome; Pediatric Obesity. 
I a prevalencia de trastornos nutricionales por exceso ha aumentado progresivamente en Chile, llegando a ser de las más altas del mundo ${ }^{1-3}$. Algo similar ha ocurrido en población infanto-juvenil ${ }^{4}$ y ha sido comunicado tanto en muestras de población de Santiago ${ }^{5,6}$ como de regiones ${ }^{7}$.

Los trastornos nutricionales por exceso en población infanto-juvenil se han asociado con anomalías metabólicas como hiperinsulinismo, intolerancia a la glucosa y dislipidemia, que junto a la hipertensión arterial conducen a la aparición precoz de enfermedad cardiovascular y diabetes mellitus tipo $2(\mathrm{DM} 2)^{8}$. El conjunto de estos factores de riesgo constituiría el síndrome metabólico (SM), condición definida para población adulta por el National Cholesterol Education Program Adults Treatment Panel (NCEP-ATP III) y adaptada para población pediátrica por Cook y cols. ${ }^{9}$. La Internacional Diabetes Federation (IDF) propuso una nueva definición de SM para niños mayores de 10 años ${ }^{10}$. La variabilidad observada en la frecuencia de SM en población infanto-juvenil dependería del criterio utilizado para su diagnóstico ${ }^{11-13}$, así como de las características de la muestra seleccionada, especialmente ruralidad y la condición socioeconómica ${ }^{14}$.

En Chile, pocos estudios han evaluado la prevalencia de SM en población infanto-juvenil. Bustos y cols. comunicaron que $37,6 \%$ de una muestra de escolares urbanos con obesidad de la Región del Biobío tenía SM mediante el criterio de Cook $^{15}$, en tanto Eyzaguirre encontró 22,7\% de SM mediante el mismo criterio en niños obesos de la ciudad de Santiago ${ }^{16}$.

En la Encuesta Nacional de Salud (ENS) 2010, la Región de la Araucanía presentaba una de las más altas prevalencias de trastornos nutricionales por exceso, DM2, dislipidemia e hipertensión arterial en población adulta ${ }^{2}$. Por otra parte, esta región tiene los peores indicadores socioeconómicos del país, una alta proporción de población rural y aborigen en algunas de sus comunas ${ }^{17,18}$. La comuna de Carahue, ubicada en la costa de la provincia de Cautín en la Región de la Araucanía, tiene una población estimada para el 2015 de 26.562 habitantes. De acuerdo a la encuesta CASEN 2013, 41,91\% de los habitantes se encontraba en situación de pobreza por ingresos y $44,9 \%$ de los hogares tenían saneamiento insuficiente, proporciones que triplican los respectivos promedios nacionales. De acuerdo al censo 2002; 29,02\% de la población comunal reconocía pertenecer al etnia mapuche ${ }^{19}$.

El propósito de este estudio fue conocer la frecuencia de trastornos nutricionales por exceso y del síndrome metabólico (SM) en escolares de la comuna de Carahue y evaluar la asociación entre resistencia a la insulina (RI), definida por dos diferentes criterios previamente descritos para población pediátrica chilena ${ }^{20,21}$ y los componentes del SM.

\section{Material y Métodos}

\section{Muestra de población y aspectos éticos}

Estudio de corte transversal que incluyó escolares de $4^{\circ}$ a $6^{\circ}$ año de enseñanza básica de la comuna de Carahue, entre noviembre de 2015 y diciembre de 2016. Los participantes del estudio fueron seleccionados mediante muestreo probabilístico aleatorio multinivel según tipo de establecimiento, ubicación geográfica y tamaño de la matrícula. El tamaño muestral se estimó considerando una matrícula de 600 estudiantes de $3^{\circ}$ a $6^{\circ}$ básico, con $40 \%$ de niños inactivos, $5 \%$ de nivel de significancia, $5 \%$ de precisión absoluta, tamaño de efecto de 1,3 y proporción de respuesta de $80 \%$ en base a proyecto previamente aprobado por el fondo de investigación UNETE (Código UNT14-008), que evaluó la misma población. Se excluyeron sujetos con enfermedad hepática o renal.

El protocolo de investigación fue aprobado por el Comité Ético Científico de la Universidad de La Frontera (Folio N026/15). Los directores de cada establecimiento firmaron una autorización para evaluar su escuela. Luego, los apoderados o cuidadores de los escolares de cursos seleccionados firmaron un consentimiento informado, para proceder con el asentimiento de los escolares para realizar los procedimientos.

\section{Evaluación clínica, medidas antropométricas y estado nutricional}

Los datos biodemográficos fueron reportados por apoderados o cuidadores y la evaluación clínica fue realizada por médico pediatra. Los participantes fueron clasificados de acuerdo a su desarrollo puberal en estadio de 1 a 5 según Tanner. Para el cálculo del índice de masa corporal (IMC), el peso y talla fueron medidos mediante balanza y estadiómetro. La circunferencia de cin- 
tura y cadera fueron medidas con cinta métrica no extensible, registrando el promedio de dos mediciones. Posteriormente se calculó el índice cintura/cadera (ICC). Las presiones arteriales sistólica y diastólica (PAS y PAD) fueron medidas con esfigmomanómetro pediátrico, registrándose el promedio de 2 mediciones consecutivas. El estado nutricional se estableció mediante el criterio percentilar del Center for Disease Control (CDC) a través del cálculo del $z$-score del IMC normalizado por edad y sexo según recomendación de la Norma para la Evaluación Nutricional de Niños, Niñas y Adolescentes de 5 a 19 años de edad del Ministerio de Salud 2016 $6^{22}$. Cuando se detectó una diferencia mayor a 1 año entre la edad cronológica y la edad biológica según su desarrollo puberal, se consideró esta última para el cálculo del IMC $z$-score. Se definió obesidad abdominal como el tener una circunferencia de cintura mayor que el percentil 90 de acuerdo a edad y sexo según la Norma para la Evaluación Nutricional de Niños, Niñas y Adolescentes de 5 a 19 años de edad del Ministerio de Salud 2016 22. Los individuos con valores de PAS y PAD superiores al percentil 90 para su edad y sexo, según recomendación del National High Blood Pressure Education Program (NHBPEP), fueron considerados con presión arterial alterada ${ }^{23}$. Finalmente, se consignó la presencia de acantosis nigricans, el perímetro del brazo y cuello. La grasa corporal fue obtenida mediante impedanciometria (Tanita TBF300, Tanita Incorporation of America, Arlington Heights, IL, EUA).

\section{Parámetros bioquímicos, cálculo de índices, definición de RI y SM}

Se obtuvieron muestras de sangre en ayuno de 10-12 h para determinar concentraciones séricas de glucosa, colesterol y triglicéridos a través de métodos enzimático-colorimétricos. La insulinemia fue determinada por quimioluminiscencia. El análisis de los parámetros bioquímicos y hormonales fueron realizadas en equipos Roche-Cobas 311 y 411 (Roche Diagnostics, Basilea, Suiza). Para describir RI se calcularon los índices HOMA-IR (Homeostatic model assessment-insulin resistance) y Quicki (Quantitative Insulin Sensitivity Check Index).

Para definir RI se utilizó HOMA-IR de acuerdo a 2 criterios propuestos para población chilena pediátrica:
1. Criterio de Burrows y cols. ${ }^{20}$ :

a. HOMA IR $>2,1$ para estadios 1 y 2 de Tanner.

b. HOMA IR $>3,3$ para estadios 3 a 5 de Tanner.

2. Criterio de Barja y cols. ${ }^{21}$ :

a. HOMA IR > 3,2 para estadios 1 y 2 de Tanner en varones $\mathrm{y}>4,1$ para estadios 1 y 2 de Tanner en mujeres.

b. HOMA IR $>4,2$ para estadios 3 a 5 de Tanner en varones y $>5,0$ para estadios 3 a 5 de Tanner en mujeres.

El SM fue definido de acuerdo a criterios modificados para población pediátrica definidos por $\mathrm{Cook}^{24}$.

\section{Análisis estadístico}

Se realizó un análisis descriptivo de los datos. La prueba de Kolmogorov-Smirnov fue usada para evaluar normalidad de variables continuas. Comparaciones entre grupos se realizaron usando pruebas de chi-cuadrado para variables categóricas y de t o ANOVA, seguido de Tukey para variables continuas con distribución normal. Variables con distribución no paramétrica fueron comparadas mediante prueba de Mann-Withney o Kruskal-Wallis seguido de método de Dunn. Para evaluar la asociación entre variables continuas se realizó un análisis de correlación lineal y cálculo del coeficiente de correlación de Pearson. Se consideró una significancia de 5\% para el análisis de los datos. Los análisis fueron realizados con el software estadísticos Minitab 17.1 (Minitab Inc. State College, PA, EUA).

\section{Resultados}

Se estudiaron 208 escolares con edad promedio de 10,4 $\pm 1,0$ años, con una relación varones/ mujeres de 49/51\%. En la Tabla 1 se resumen las variables clínicas y antropométricas de la muestra según estado nutricional; $71 \%$ de los escolares tenían trastornos nutricionales por exceso (37,9\% sobrepeso y $33,1 \%$ obesidad), siendo que esta condición fue más frecuente en el sexo femenino $(\mathrm{p}=0,030)$. La PAS, así como la frecuencia de presión arterial alterada, fueron mayores en el grupo con obesidad que en los eutróficos $(p<0,05)$. Se observó mayor frecuencia de acantosis nigricans en el grupo obeso que en los otros grupos. 
Tabla 1. Datos clínicos, biodemográficos y antropométricos de acuerdo al estado nutricional en escolares de la comuna de Carahue

\begin{tabular}{|c|c|c|c|c|c|}
\hline Variable & $\begin{array}{l}\text { Grupo total } \\
(n=208)\end{array}$ & $\begin{array}{l}\text { Normopeso } \\
(n=60)\end{array}$ & $\begin{array}{c}\text { Sobrepeso } \\
(n=79)\end{array}$ & $\begin{array}{l}\text { Obesos } \\
(n=69)\end{array}$ & Valor $\mathbf{p}$ \\
\hline \multicolumn{6}{|c|}{ Datos clínicos y biodemográficos } \\
\hline Edad, años & $10,4 \pm 1,0$ & $10,3 \pm 1,02$ & $10,4 \pm 0,96$ & $10,4 \pm 1,10$ & 0,561 \\
\hline Estadio Tanner (1-2/3-5), \% & $66 / 34$ & $60 / 40$ & $63 / 37$ & $75 / 25$ & 0,152 \\
\hline $\operatorname{Sexo}(M / F), \%$ & $49 / 51$ & $35 / 65$ & $51 / 49$ & $58 / 42$ & 0,030 \\
\hline Acantosis nigricans & $8,7 \%(18)$ & $0 \%(0)$ & $1 \%(1)$ & $25 \%(17)$ & $<0,001$ \\
\hline PAS, $\mathrm{mmHg}$ & $109,6 \pm 18,6$ & $105,2 \pm 18,4^{a}$ & $107,6 \pm 18,3^{a b}$ & $115,7 \pm 18,1^{b}$ & 0,013 \\
\hline $\mathrm{PAD}, \mathrm{mmHg}$ & $61,5 \pm 16,7$ & $63,0 \pm 17,8$ & $57,8 \pm 13,8$ & $64,4 \pm 18,3$ & 0,091 \\
\hline Presión arterial alterada & $35 \%(55)$ & $29 \%(13)$ & $29 \%(17)$ & $49 \%(25)$ & 0,048 \\
\hline Síndrome metabólico & $12 \%(24)$ & $0 \%(0)$ & $0 \%(0)$ & $38 \%(24)$ & $<0,001$ \\
\hline \multicolumn{6}{|c|}{ Parámetros antropométricos } \\
\hline Peso, $\mathrm{Kg}$ & $46,6 \pm 10,9$ & $37,8 \pm 6,9^{a}$ & $44,8 \pm 7,4^{b}$ & $56,2 \pm 9,7^{c}$ & $<0,001$ \\
\hline IMC z-score & $1,50 \pm 0,97$ & $0,36 \pm 0,55^{a}$ & $1,42 \pm 0,29^{b}$ & $2,58 \pm 0,40^{c}$ & $<0,001$ \\
\hline Obesidad abdominal & $26 \%(54)$ & $2 \%(1)$ & $8 \%(6)$ & $26 \%(47)$ & $<0,001$ \\
\hline $\mathrm{CA}, \mathrm{cm}$ & $71,9 \pm 9,7$ & $63,0 \pm 5,4^{a}$ & $70,4 \pm 5,8^{b}$ & $81,2 \pm 7,9$ & $<0,001$ \\
\hline ICC & $0,85 \pm 0,08$ & $0,82 \pm 0,05^{a}$ & $0,85 \pm 0,04^{b}$ & $0,89 \pm 0,05^{c}$ & $<0,001$ \\
\hline Circunferencia brazo, cm & $22,6 \pm 2,8$ & $20,5 \pm 1,9^{a}$ & $22,3 \pm 2,0^{b}$ & $25,1 \pm 2,4^{c}$ & $<0,001$ \\
\hline Perímetro cervical, $\mathrm{cm}$ & $30,9 \pm 2,6$ & $28,6 \pm 2,1^{a}$ & $30,6 \pm 1,58^{b}$ & $33,1 \pm 2,01^{c}$ & $<0,001$ \\
\hline Grasa corporal, \% & $28,4 \pm 8,7$ & $21,7 \pm 6,8^{a}$ & $27,3 \pm 5,7^{b}$ & $36,4 \pm 7,1^{c}$ & $<0,001$ \\
\hline
\end{tabular}

Número de individuos en paréntesis. Variables categóricas son presentadas como porcentaje y comparadas por prueba de $\chi^{2}$. Variables continuas son presentadas como media \pm desvío estándar y fueron comparadas por ANOVA seguido de Tukey para variables con distribución paramétrica o usando Kruskall-Wallis seguido de método de comparación de Dunnett en el caso de variables no paramétricas. PAS: Presión arterial sistólica, PAD: Presión arterial diastólica, IMC: Índice de masa corporal, CA: Circunferencia abdominal, ICC: Índice cintura/cadera. a,b,c: Letras diferentes en la misma línea representan diferencia estadísticamente significativa.

El 12\% de la muestra estudiada reunió criterios para SM, pesquisándose esta condición exclusivamente en el grupo con obesidad (38\%; $\mathrm{p}<0,001$; Tabla 1). También se observó en las variables analíticas bioquímicas un perfil de mayor riesgo cardiometabólico en este grupo, con valores significativamente aumentados de triglicéridos, colesterol VLDL, colesterol no HDL, insulinemia, HOMA-IR y Quicki, así como valores reducidos de colesterol HDL ( $p<0,05$; Tabla 2$)$.

La frecuencia de RI en la muestra fue $51 \%$ y 19\% según los criterios de Burrows y Barja, respectivamente. La frecuencia de RI no varió significativamente según el sexo o desarrollo puberal, independientemente del criterio utilizado, sin embargo, esta fue más frecuente en individuos con sobrepeso y obesidad $(p<0,001)$ y en aquellos con obesidad abdominal ( $\mathrm{p}<0,001$; Tabla 3$)$.

Todas las medidas antropométricas evaluadas presentaron valores aumentados en niños con RI según criterio de Barja $(\mathrm{p}<0,05)$, con el criterio de Burrows no hubo diferencia estadística en el valor de ICC (Tabla 4). Al ser evaluados los parámetros del perfil bioquímico, los individuos con RI para ambos criterios presentaron valores significativamente aumentados de triglicéridos, colesterol VLDL, glucosa e insulina, así como los índices HOMA-IR y Quicki (Tabla 4).

En la Tabla 5 se aprecia que el SM fue más frecuente en individuos con RI, independientemente del criterio utilizado, sin embargo, la diferencia de la frecuencia de los componentes del SM entre sujetos resistentes y sensibles a la insulina fue mucho mayor cuando se utilizó el criterio de Barja (Tabla 5). También se observaron diferencias en la frecuencia de los componentes del SM por separado, cuya magnitud también varió de acuerdo al criterio de RI.

En la Tabla 6 se muestra la correlación entre la insulinemia e índices de sensibilidad insulínica con parámetros antropométricos. Con excepción del ICC, todos los parámetros antropométicos se correlacionaron positiva y significativamente con los valores de insulina y HOMA-IR; y negativamente con los valores de Quicki. Entre las medidas antropométricas analizadas, la circunferencia de brazo presentó la correlación más fuerte con los parámetros de RI. 
Tabla 2. Parámetros bioquímicos y hormonales de acuerdo al estado nutricional en escolares de la comuna de Carahue

\begin{tabular}{|lccccc|}
\hline Variable & $\begin{array}{c}\text { Grupo total } \\
(\mathbf{n}=\mathbf{2 0 8})\end{array}$ & $\begin{array}{c}\text { Normopeso } \\
(\mathbf{n}=\mathbf{6 0})\end{array}$ & $\begin{array}{c}\text { Sobrepeso } \\
\mathbf{( n = 7 9 )}\end{array}$ & $\begin{array}{c}\text { Obesos } \\
(\mathbf{n}=\mathbf{6 9})\end{array}$ & Valor p \\
\hline Colesterol total, $\mathrm{mg} / \mathrm{dL}$ & $154,0 \pm 28,9$ & $150,3 \pm 31,6$ & $154,0 \pm 27,3$ & $157,4 \pm 28,4$ & 0,392 \\
\hline Triglicéridos, $\mathrm{mg} / \mathrm{dL}$ & $99,4 \pm 68,2$ & $79,5 \pm 31,4^{\mathrm{a}}$ & $90,2 \pm 37,4^{\mathrm{a}}$ & $127,3 \pm 102,4^{\mathrm{b}}$ & $<0,001$ \\
\hline Colesterol LDL, mg/dL & $84,0 \pm 23,85$ & $79,0 \pm 24,8$ & $84,7 \pm 23,0$ & $86,9 \pm 23,9$ & 0,070 \\
\hline Colesterol HDL, mg/dL & $50,5 \pm 13,4$ & $55,4 \pm 13,7^{\mathrm{a}}$ & $51,2 \pm 11,9^{\mathrm{a}}$ & $45,8 \pm 13,4^{\mathrm{b}}$ & $<0,001$ \\
\hline Colesterol no-HDL, mg/dL & $103,5 \pm 28,0$ & $94,9 \pm 26,7^{\mathrm{a}}$ & $102,8 \pm 25,4^{\mathrm{a}, \mathrm{b}}$ & $111,7 \pm 30,1^{\mathrm{b}}$ & 0,002 \\
\hline Colesterol VLDL, mg/dL & $19,9 \pm 13,7$ & $15,8 \pm 6,3^{\mathrm{a}}$ & $18,0 \pm 7,5^{\mathrm{a}}$ & $25,5 \pm 20,5^{\mathrm{b}}$ & $<0,001$ \\
\hline Glucosa, $\mathrm{mg} / \mathrm{dL}$ & $85,3 \pm 7,3$ & $84,8 \pm 6,1$ & $85,3 \pm 6,3$ & $85,6 \pm 9,0$ & 0,655 \\
\hline Insulina, $\mu \mathrm{U} / \mathrm{mL}$ & $14,1 \pm 9,4$ & $10,1 \pm 5,5^{\mathrm{a}}$ & $13,5 \pm 8,2^{\mathrm{b}}$ & $18,0 \pm 11,6^{\mathrm{c}}$ & $<0,001$ \\
\hline HOMA-IR & $3,00 \pm 2,18$ & $2,15 \pm 1,25^{\mathrm{a}}$ & $2,86 \pm 1,82^{\mathrm{a}}$ & $3,87 \pm 2,81^{\mathrm{b}}$ & $<0,001$ \\
\hline Quicki & $0,337 \pm 0,033$ & $0,353 \pm 0,037^{\mathrm{a}}$ & $0,336 \pm 0,029^{\mathrm{b}}$ & $0,323 \pm 0,027^{\mathrm{b}}$ & $<0,001$ \\
\hline
\end{tabular}

Valores son presentadas como media \pm desvío estándar y fueron comparadas por ANOVA seguido de Tukey para variables con distribución paramétrica o usando Kruskall-Wallis seguido de método de comparación de Dunnett en el caso de variables no paramétricas. LDL: lipoproteína de baja densidad, HDL: lipoproteína de alta densidad, VLDL: lipoproteína de muy baja densidad, HOMA-IR: Homeostatic model assessment-insulin resistance, Quicki: Quantitative Insulin Sensitivity Check Index. a,b,c: Letras diferentes en la misma línea representan diferencia estadísticamente significativa.

\section{Tabla 3. Prevalencia de resistencia a la insulina en escolares de la comuna de Carahue}

\begin{tabular}{|c|c|c|}
\hline & \multicolumn{2}{|c|}{ Resistencia a la insulina } \\
\hline & Criterio 1 (Burrows, 2006) & Criterio 2 (Barja, 2011) \\
\hline Grupo total, \% (n) & $51 \%(105)$ & $19 \%(39)$ \\
\hline \multicolumn{3}{|l|}{ Sexo, \% (n) } \\
\hline Masculino & $50 \%(50)$ & $23 \%(23)$ \\
\hline \multirow[t]{2}{*}{ Femenino } & $54 \%(56)$ & $15 \%(16)$ \\
\hline & $\chi^{2}=0,387 ; G L=1 ; p=0,534$ & $\chi^{2}=1,815 ; G L=1 ; p=0,178$ \\
\hline \multicolumn{3}{|l|}{ Desarrollo puberal, \% (n) } \\
\hline Tanner 1-2 & $56 \%(75)$ & $22 \%(29)$ \\
\hline \multirow[t]{2}{*}{ Tanner 3-5 } & $44 \%(30)$ & $13 \%(9)$ \\
\hline & $\chi^{2}=2,372 ; G L=1 ; p=0,124$ & $\chi^{2}=2,021 ; G L=1 ; p=0,155$ \\
\hline \multicolumn{3}{|l|}{ Estado nutricional, \% (n) } \\
\hline Normopeso & $32 \%(18)$ & $5 \%(3)$ \\
\hline Sobrepeso & $48 \%(38)$ & $15 \%(12)$ \\
\hline \multirow[t]{2}{*}{$(n=68)$} & $72 \%(49)$ & $35 \%(24)$ \\
\hline & $\chi^{2}=21,562 ; G L=2 ; p<0,001$ & $\chi^{2}=19,372 ; G L=2 ; p<0,001$ \\
\hline \multicolumn{3}{|l|}{ Obesidad abdominal, \% (n) } \\
\hline Circunferencia cintura <p90 $(n=150)$ & $43 \%(65)$ & $12 \%(18)$ \\
\hline \multirow[t]{2}{*}{ Circunferencia cintura $>$ p90 $(n=54)$} & $74 \%(40)$ & $39 \%(21)$ \\
\hline & $\chi^{2}=15,552 ; G L=1 ; p<0,001$ & $\chi^{2}=18,566 ; G L=1 ; p<0,001$ \\
\hline
\end{tabular}

Datos de son presentados en porcentaje de individuos resistentes a la insulina de acuerdo a los criterios descritos por Burrows et al. 2006 (Criterio 1) y Barja et al. 2011 (Criterio 2). Comparaciones de la prevalencia de resistencia insulínica de acuerdo al sexo, desarrollo puberal, estado nutricional y obesidad abdominal fueron realizadas mediante un análisis de chi-cuadrado $\left(\chi^{2}\right)$. GL: Grados de libertad. 
Tabla 4. Parámetros antropométricos y bioquímicos en individuos resistentes y sensibles a la insulina de la comuna de Carahue

\begin{tabular}{|c|c|c|c|c|c|c|}
\hline \multirow[b]{3}{*}{ Variable } & \multicolumn{6}{|c|}{ Resistencia a la insulina } \\
\hline & \multicolumn{3}{|c|}{ Criterio 1 (Burrows, 2006) } & \multicolumn{3}{|c|}{ Criterio 2 (Barja, 2011) } \\
\hline & Resistente & Sensible & Valor $\mathbf{p}$ & Resistente & Sensible & Valor $p$ \\
\hline \multicolumn{7}{|c|}{ Parámetros antropométricos } \\
\hline Peso, $\mathrm{Kg}$ & $50,3 \pm 11,1$ & $42,7 \pm 9,3$ & $<0,001$ & $54,6 \pm 11,7$ & $44,8 \pm 9,8$ & $<0,001$ \\
\hline IMC z-score & $1,86 \pm 0,87$ & $1,14 \pm 0,94$ & $<0,001$ & $2,23 \pm 0,83$ & $1,34 \pm 0,93$ & $<0,001$ \\
\hline $\mathrm{CA}, \mathrm{cm}$ & $74,9 \pm 9,8$ & $68,9 \pm 8,5$ & $<0,001$ & $78,6 \pm 10,5$ & $70,4 \pm 8,8$ & $<0,001$ \\
\hline ICC & $0,86 \pm 0,05$ & $0,85 \pm 0,05$ & 0,310 & $0,87 \pm 0,06$ & $0,85 \pm 0,05$ & 0,038 \\
\hline Obesidad abdominal & $38 \%(40)$ & $14 \%(14)$ & $<0,001$ & $54 \%(21)$ & $20 \%(33)$ & $<0,001$ \\
\hline Circunferencia brazo, $\mathrm{cm}$ & $23,8 \pm 2,9$ & $21,5 \pm 2,3$ & $<0,001$ & $24,7 \pm 2,8$ & $22,2 \pm 2,6$ & $<0,001$ \\
\hline Perímetro cervical, $\mathrm{cm}$ & $31,7 \pm 2,6$ & $30,1 \pm 2,3$ & $<0,001$ & $32,6 \pm 2,1$ & $30,5 \pm 2,5$ & $<0,001$ \\
\hline Grasa corporal, \% & $31,1 \pm 8,5$ & $25,9 \pm 8,4$ & $<0,001$ & $34,2 \pm 8,6$ & $27,3 \pm 8,4$ & 0,001 \\
\hline \multicolumn{7}{|l|}{ Parámetros bioquímicos } \\
\hline Colesterol total, mg/DI & $156,3 \pm 27,3$ & $151,5 \pm 30,1$ & 0,237 & $154,0 \pm 21,4$ & $153,7 \pm 30,0$ & 0,939 \\
\hline Triglicéridos, mg/dL & $113,8 \pm 85,8$ & $84,0 \pm 36,5$ & $<0,001$ & $137,8 \pm 121,4$ & $90,4 \pm 44,0$ & $<0,001$ \\
\hline Colesterol LDL, mg/dL & $84,5 \pm 22,8$ & $83,5 \pm 24,8$ & 0,894 & $80,1 \pm 16,0$ & $84,9 \pm 25,1$ & 0,315 \\
\hline Colesterol HDL, mg/dL & $49,9 \pm 13,8$ & $51,2 \pm 12,9$ & 0,349 & $48,1 \pm 12,3$ & $51,1 \pm 13,6$ & 0,490 \\
\hline Colesterol no-HDL, mg/dL & $106,4 \pm 27,9$ & $100,3 \pm 27,9$ & 0,129 & $105,6 \pm 23,2$ & $103,0 \pm 29,1$ & 0,449 \\
\hline Colesterol VLDL, mg/dL & $22,8 \pm 17,2$ & $16,8 \pm 7,4$ & $<0,001$ & $27,6 \pm 24,3$ & $18,1 \pm 8,8$ & $<0,001$ \\
\hline Glucosa, mg/dL & $86,7 \pm 8,4$ & $83,7 \pm 5,5$ & 0,002 & $88,6 \pm 7,6$ & $84,5 \pm 6,9$ & 0,002 \\
\hline Insulina, $\mu \mathrm{U} / \mathrm{mL}$ & $19,7 \pm 9,8$ & $8,1 \pm 3,0$ & $<0,001$ & $27,8 \pm 11,7$ & $10,8 \pm 4,7$ & $<0,001$ \\
\hline HOMA-IR & $4,24 \pm 2,37$ & $1,67 \pm 0,66$ & $<0,001$ & $6,14 \pm 2,94$ & $2,26 \pm 1,00$ & $<0,001$ \\
\hline Quicki & $0,314 \pm 0,017$ & $0,360 \pm 0,028$ & $<0,001$ & $0,298 \pm 0,013$ & $0,345 \pm 0,029$ & $<0,001$ \\
\hline
\end{tabular}

Número de individuos en paréntesis. Resistentes a la insulina fue definida de acuerdo a los criterios descritos por Burrows et al. 2006 (Criterio 1) y Barja et al. 2011 (Criterio 2). Variables categóricas son presentadas como porcentaje y comparadas por prueba de chi-cuadrado. Variables continuas son presentadas como media \pm desvío estándar y fueron comparadas por prueba de t o Mann-Whitney para variables con distribución paramétrica o no paramétricas, respectivamente. IMC: Índice de masa corporal, CA: Circunferencia abdominal, ICC: Índice cintura/cadera, LDL: lipoproteína de baja densidad, HDL: lipoproteína de alta densidad, VLDL: lipoproteína de muy baja densidad, HOMA-IR: Homeostatic model assessment-insulin resistance, Quicki: Quantitative Insulin Sensitivity Check Index.

Tabla 5. Relación de resistencia a la insulina con componentes para el diagnóstico de síndrome metabólico en escolares de la comuna de Carahue

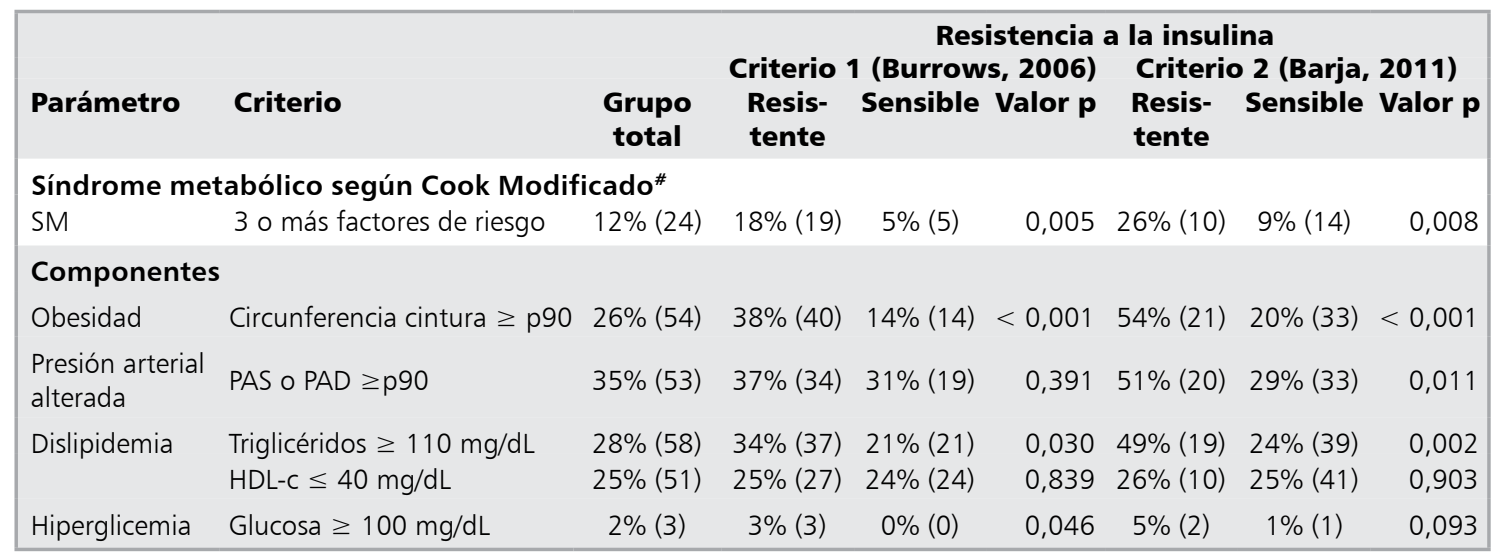

\#Definición de Síndrome metabólico de acuerdo a criterio modificado descrito por Cook et al. ${ }^{24}$. Datos de son presentados en porcentaje de individuos resistentes a la insulina de acuerdo a los criterios descritos por Burrows et al. 2006 (Criterio 1) y Barja et al. 2011 (Criterio 2). Comparaciones de la prevalencia de SM o de cada uno de sus componentes de acuerdo al estado de resistente o sensible a la insulina fueron realizadas mediante un análisis de chi-cuadrado $\left(\chi^{2}\right)$. El número de individuos se encuentra entre paréntesis. GL: PAS: Presión arterial sistólica; PAD: Presión arterial diastólica; HDL-c: Colesterol de la lipoproteína de alta densidad. 
Tabla 6. Correlación de insulinemia e índices de sensibilidad insulínica con parámetros antropométricos en escolares de la comuna de Carahue

\begin{tabular}{|lcccccc|}
\hline Parámetro & \multicolumn{2}{c}{ Insulinemia } & \multicolumn{2}{c}{ HOMA-IR } & \multicolumn{2}{c|}{ Quicki } \\
\hline Peso & $\mathbf{R}$ & Valor $\mathbf{p}$ & $\mathbf{R}$ & Valor $\mathbf{p}$ & R & Valor $\mathbf{p}$ \\
\hline IMC z-score & 0,461 & $<0,001$ & 0,451 & $<0,001$ & $-0,548$ & $<0,001$ \\
\hline CA & 0,386 & $<0,001$ & 0,360 & $<0,001$ & $-0,416$ & $<0,001$ \\
ICC & 0,372 & $<0,001$ & 0,357 & $<0,001$ & $-0,446$ & $<0,001$ \\
Circunferencia brazo & 0,562 & $<0,001$ & 0,560 & $<0,001$ & $-0,556$ & $<0,001$ \\
Perímetro cervical & 0,349 & $<0,001$ & 0,338 & $<0,001$ & $-0,452$ & $<0,001$ \\
\hline Grasa corporal & 0,447 & $<0,001$ & 0,410 & $<0,001$ & $-0,459$ & $<0,001$ \\
\hline
\end{tabular}

r: Coeficiente de correlación de Pearson. IMC: Índice de masa corporal, CA: Circunferencia abdominal, ICC: Índice cintura/cadera, HOMA-IR: Homeostatic model assessment-insulin resistance, Quicki: Quantitative Insulin Sensitivity Check Index.

\section{Discusión}

En nuestra muestra de escolares de la comuna de Carahue encontramos una mayor frecuencia de obesidad que el estudio de Bustos en individuos de comunas urbanas de la Región del Biobío el año 2009 (33,1\% vs 9,2\% $)^{15}$. La proporción de individuos obesos con SM fue 38\% en nuestro estudio y $37,5 \%$ en el de Bustos. En cambio, Eyzaguirre reportó solo $22,7 \%$ de SM en una muestra de individuos obesos que consultaron en una clínica privada de la ciudad de Santiago ${ }^{16}$. La mayor proporción de individuos obesos en nuestro estudio podría atribuirse al aumento progresivo en la prevalencia de trastornos nutricionales por exceso en el tiempo que separa a nuestro estudio de los otros. Por otra parte, la diferencia en la proporción de individuos obesos con SM de nuestro estudio respecto a la de Eyzaguirre podría ser explicada por diferencias principalmente socioeconómicas y menos probablemente étnicas ${ }^{19,25-26}$. Nuestros resultados, al igual que los de Bustos y Eyzaguirre, muestran una clara asociación entre el peso y los parámetros bioquímicos que expresan riesgo cardiometabólico.

La RI, clínicamente definida como un estado donde la insulina produce una respuesta disminuida en su acción glucoregulatoria, es claramente reconocida como un factor de riesgo para DM2 Sin embargo, no existen criterios ampliamente aceptados para su diagnóstico, por lo cual el concepto de RI debe ser cuidadosamente interpretado en pediatría $^{27}$. La técnica de referencia o estándar de oro para determinar sensibilidad a la insulina es el clamp hiperinsulinémico-hiperglicémico que tiene escasa aplicación clínica debido a su complejidad e invasividad ${ }^{28}$. Entre los métodos alternativos, los índices HOMA-IR y Quicki presentan una moderada a buena correlación con el clamp, presentando coeficientes de correlación que van de $-0,49$ a - $-0,91$ en el caso del HOMA-IR y de 0,43 a 0,91 para Quicki ${ }^{27}$.

En Chile, se han propuesto 2 criterios para el diagnóstico de RI en población pediátrica. Nuestros resultados sugieren que el criterio de Barja $^{21}$, si bien reconoce una proporción menor de sujetos con RI que el criterio de Burrows ${ }^{20}$, tiende a identificar mejor aquellos con SM o sus componentes, probablemente debido a que dicho estudio estableció valores de corte para RI a partir de escolares eutróficos, normalizados según sexo y desarrollo puberal, excluyendo individuos con glicemia alterada.

El II Consenso de la Sociedad Chilena de Endocrinología y Diabetes sobre RI, comunicado en $2015^{29}$, no recomienda la medición de insulina en la práctica clínica, considerando las limitaciones del método y la falta de validación para población pediátrica. Nuestros resultados muestran que $72 \%$ de los escolares con obesidad tienen hiperinsulinismo, según criterio de Burrows, pero solo $18 \%$ de estos califican para SM. Al utilizar el criterio de Barja, solo 35\% de los escolares obesos tenía hiperinsulinismo, pero de estos $26 \%$ calificó para SM. Si bien la alta prevalencia de hiperinsulinismo y su escasa contribución en la 
conducta terapéutica refuerzan la recomendación del citado consenso de diagnosticar hiperinsulinismo por los hallazgos clínicos y bioquímicos, la caracterización de la población infanto-juvenil de acuerdo a índices de resistencia a la insulina y su correlación con parámetros antropométricos y bioquímicos son importantes para identificar factores de riesgo cardiometabólico y su relación con el desarrollo de enfermedad cardiovascular en nuestra población.

Otro hallazgo de nuestro estudio fue la alta correlación entre los perímetros del brazo y cervical de los escolares con el grado de RI, lo que podría permitir utilizar estos parámetros antropométricos como predictores de riesgo cardiometabólico en esta muestra de escolares.

La principal limitación de nuestros resultados es la poca confiabilidad de algunas variables sociodemográficas como ruralidad y etnia. Por otro lado, algunas de las definiciones, tales como el diagnóstico de SM y RI, carecen de una validación amplia en población pediátrica, lo cual es una debilidad transversal de trabajos que evalúan individuos en este rango etario. A pesar de que el criterio para SM de la IDF para población pediátrica es ampliamente utilizado ${ }^{10}$, nuestro estudio consideró la definición de Cook modificado ${ }^{24}$, debido al rango etario de la población de estudio (el criterio de IDF es definido solo para niños mayores de 10 años) y por la comparabilidad con otros trabajos que evaluaron SM en nuestra población. En este sentido, los valores de corte propuestos por el estudio de Barja como criterio de RI en población pediátrica ${ }^{21}$, si bien son un importante avance para la caracterización de población pediátrica chilena, necesitan ser validados en otras poblaciones de nuestro país, siendo este estudio una contribución en este sentido. Otra debilidad del presente trabajo es la dificultad de asegurar el ayuno en este tipo de población, lo cual se intentó minimizar a través de reuniones informativas con los responsables de los menores que acompañaron las evaluaciones.

En conclusión, una proporción mayoritaria de nuestra muestra de escolares de la comuna de Carahue tiene trastornos nutricionales por exceso, con una proporción de obesidad mucho mayor que la comunicada previamente. La frecuencia de SM entre los individuos obesos no ha cambiado respecto a lo encontrado en una población comparable. En futuras investigaciones debemos iden- tificar los factores que explican la alta frecuencia de obesidad en esta población.

Agradecimientos: Agradecemos a todos los participantes y funcionarios de escuelas y Departamento de Educación Municipal de la comuna de Carahue que voluntariamente contribuyeron con esta investigación. El presente trabajo fue financiado por el Fondo de Investigación UNETE (\#UNT15-004) del Convenio de Desempeño Regional, FRO 1301, Universidad de La Frontera y proyecto Fondecyt 11150445.

\section{Referencias}

1. ENS Chile 2003,disponible en: http://bibliotecaminsal-chile.bvsalud.org/cgi-bin/wxis1660.exe/lildbi/iah/

2. ENS Chile 2010, disponible en: http://web.minsal.cl/ portal/url/item/bcb03d7bc28b64dfe040010165012d23. pdf

3. ENS Chile 2016, primeros resultados, disponible en: http://web.minsal.cl/wp-content/uploads/2017/11/ENS2016-17_PRIMEROS-RESULTADOS.pdf

4. Rivera JA, de Cossío TG, Pedraza LS, Aburto TC, Sánchez TG, Martorell R. Childhood and adolescent overweight and obesity in Latin America: a systematic review. Lancet Diabetes Endocrinol 2014; 2: 321-32.

5. Kain J, Uauy R, Vio F, Albala C. Trends in overweight and obesity prevalence in Chilean children: comparison of three definitions. Eur J Clin Nutr 2002; 56: 200-4.

6. Amigo H, Bustos P, Erazo M, Cumsille P, Silva C. Factores determinantes del exceso de peso en escolares: Un estudio multinivel. Rev Med Chile 2007; 135: 1510-8.

7. Ulloa N, Sapunar J, Bustos P, Sáez K, Asenjo S, Taibo $\mathrm{M}$, et al. Epidemiología del sobrepeso y obesidad infanto-juvenil en las comunas de Concepción, Coronel y Hualpén, VIII Región de Chile. Rev Med Chile 2010; 138: 1365-72.

8. Aggoun Y. Obesity, metabolic syndrome, and cardiovascular disease. Pediatr Res 2007; 61: 653-9.

9. Cook S, Weitzman M, Auinger P, Nguyen M, Dietz WH. Prevalence of a metabolic síndrome phenotype in adolescents: findings from the third National Health and Nutrition Examination Survey, 1988-1994. Arch Pediatr Adolesc Med 2003; 157: 821-7.

10. Zimmet P, Alberti KG, Kaufman F Tajima N, Silink M, Arslanian S, et al; IDF Consensus Group. The metabolic syndrome in children and adolescents - an IDF consensus report. Pediatr Diabetes 2007; 8: 299-306.

11. Cook S, Auinger P, Li C, Ford ES. Metabolic syndrome rates in United States adolescents, from the National 
Health and Nutrition Examination Survey, 1999-2002. J Pediatr 2008; 152 (2): 165-70.

12. Sen Y, Kandemir N, Alikasifoglu A, Gonc N, Ozon A. Prevalence and risk factors of metabolic syndrome in obese children and adolescents: the role of the severity of obesity. Eur J Pediatr 2008; 167: 1183-9.

13. Han JC, Lawlor DA, Kimm SY. Childhood obesity. Lancet 2010; 375: 1737-48.

14. Johnson JA 3rd, Johnson AM. Urban-rural differences in childhood and adolescent obesity in the United States: a systematic review and meta-analysis. Child Obes 2015; 11: 233-41.

15. Bustos P, Sáez K, Gleisner A, Ulloa N, Calvo C, Asenjo S. Metabolic syndrome in obese adolescents. Pediatr Diabetes 2010; 11: 55-60.

16. Eyzaguirre F, Silva R, Román R, Palacio A, Cosentino M, Vega V, García H. Prevalencia de síndrome metabólico en niños y adolescentes que consultan por obesidad. Rev Med Chile 2011; 139: 732-8.

17. Disponible en: http://www.ministeriodesarrollosocial. gob.cl/pdf/upload/Informe_de_Desarrollo_Social_2016. pdf

18. Disponible en: http://www.ine.cl/docs/default-source/ FAQ/s\%C3\%ADntesis-de-resultados-censo-2002.pdf?sfvrsn $=2$

19. Disponible en: http://reportescomunales.bcn.cl/2015/ index.php/Carahue

20. Burrows AR, Leiva BL, Burgueño AM, Maggi MA, Giadrosic RV, Díaz BE, et al. [Insulin sensitivity in children aged 6 to 16 years: association with nutritional status and pubertal development]. Rev Med Chile 2006; 134: 1417-26.
21. Barja S, Arnaiz P, Domínguez A, Villarroel L, Cassis B, Castillo O, et al. [Normal plasma insulin and HOMA values among Chilean children and adolescents]. Rev Med Chile 2011; 139: 1435-43.

22. Disponible en: https://es.scribd.com/document/ 319272914/Norma-Para-La-Evaluacion-Nutricionalde-Ninos-y-Adolescentes-de-5-a-19-Anos-2016.

23. National High Blood Pressure Education Program Working Group on High Blood Pressure in Children and Adolescents. The fourth report on the diagnosis, evaluation, and treatment of high blood pressure in children and adolescents. Pediatrics 2004; 114 (2 Suppl 4th Report): 555-76.

24. Cook S, Weitzman M, Auinger P, Nguyen M, Dietz WH. Prevalence of a metabolic syndrome phenotype in adolescents: findings from the third National Health and Nutrition Examination Survey, 1988-1994. Arch Pediatr Adolesc Med 2003; 157: 821-7.

25. Disponible en: http://reportescomunales.bcn.cl/2015/ index.php/Coronel

26. Disponible en: http://reportescomunales.bcn.cl/2015/ index.php/Las_Condes

27. Brown RJ, Yanovski JA. Estimation of insulin sensitivity in children. Pediatrics Diabetes 2014; 15: 151-61.

28. Levy-Marchal C, Arnslanian S, Cutfield W, Sinaiko A, Druet $\mathrm{C}$, Marcovecchio $\mathrm{M}$, et al. Insulin resistance in children: consensus, perspective, and future directions. J Clin Endocrinol Metab 2010; 95: 5189-98.

29. Pollak F, Araya V, Lanas A, Sapunar J, Arrese M, Aylwin CG, et al. II Consenso de la Sociedad Chilena de Endocrinología y Diabetes sobre resistencia a la insulina. Rev Med Chile 2015; 143: 637-50. 